\title{
Erratum
}

\section{Lifetime of Pair Excitations in Nuclear Fission}

G. Schütte

Z. Physik A288, 161-166 (1978)

Formula (3.14) must read:

$W=|c|^{2} e^{-2 \Gamma\left(t-t_{1}\right)} \frac{|d|^{2 N} e^{2 \Gamma\left(t-t_{1}\right)}-1}{|d|^{2} e^{2 \Gamma \tau}-1} \cdot+|d|^{2 N}$.

The interval $\tau$ is an average value of $t_{i+1}-t_{i}$.

G. Schütte

Institut für Theoretische Physik

Universität Heidelberg

Philosophenweg 19

D-6900 Heidelberg 1

Federal Republic of Germany

Responsible for advertisements: L. Siegel, Kurfürstendamm 237, D-1000 Berlin 15, Telephone: (030)8821031, Telex: 01-85411 - Springer-Verlag Berlin . Heidelberg · New York Printers: Universitätsdruckerei H. Stïrtz AG, Würzburg - Printed in Germany - $\mathcal{O}$ by Springer-Verlag Berlin Heidelberg 1979 